\title{
openheart Simple renal cysts and bovine aortic arch: markers for aortic disease
}

\author{
Adam J Brownstein, ${ }^{1,2}$ Syed Usman Bin Mahmood, ${ }^{1}$ Ayman Saeyeldin, ${ }^{1}$ \\ Camilo Velasquez Mejia, ${ }^{1}$ Mohammad A Zafar, ${ }^{1}$ Yupeng $\mathrm{Li}^{3}{ }^{3}$ John A Rizzo, ${ }^{1,4,5}$ \\ Neera K Dahl, ${ }^{6}$ Young Erben, ${ }^{1,7}$ Bulat A Ziganshin, ${ }^{1,8}$ John A Elefteriades ${ }^{1}$
}

\begin{abstract}
- Additional material is published online only. To view please visit the journal online (http://dx.doi.org/10.1136/ openhrt-2018-000862).
\end{abstract}

To cite: Brownstein AJ Bin Mahmood SU, Saeyeldin A, et al. Simple renal cysts and bovine aortic arch: markers for aortic disease. Open Heart 2019;6:e000862. doi:10.1136/ openhrt-2018-000862

Received 22 May 2018 Revised 26 June 2018 Accepted 3 July 2018
Check for updates

(c) Author(s) (or their employer(s)) 2019. Re-use permitted under CC BY-NC. No commercial re-use. See rights and permissions. Published by BMJ.

For numbered affiliations see end of article.

Correspondence to Dr Adam J Brownstein; abrowns3@jhmi.edu

\section{ABSTRACT}

Objective This study aimed to assess the prevalence of thoracic aortic disease (TAD) and abdominal aortic aneurysms (AAA) among patients with simple renal cyst (SRC) and bovine aortic arch (BAA).

Methods Through a retrospective search for patients who underwent both chest and abdominal CT imaging at our institution from 2012 to 2016, we identified patients with SRC and BAA and propensity score matched them to those without these features by age, gender and presence of hypertension, hyperlipidaemia, diabetes and chronic kidney disease.

Results of a total of 35498 patients, 6366 were found to have SRC. Compared with the matched population without SRC, individuals with SRC were significantly more likely to have TAD (10.1\% vs $3.9 \%)$, ascending aortic aneurysm $(8.0 \%$ vs $3.2 \%)$, descending aortic aneurysm (3.3\% vs $0.9 \%$ ), type A aortic dissection ( $0.6 \%$ vs $0.2 \%)$, type $B$ aortic dissection (1.1\% vs $0.3 \%$ ) and AAA $(7.9 \%$ vs $3.3 \%$ ). The 920 patients identified with BAA were significantly more likely to have TAD ( $21.8 \%$ vs $4.5 \%)$, ascending aortic aneurysm (18.4\% vs $3.2 \%)$, descending aortic aneurysm (6.5\% vs $2.0 \%)$, type $A$ aortic dissection ( $1.4 \%$ vs $0.4 \%)$ and type $B$ aortic dissection ( $2.4 \%$ vs $0.7 \%$ ) than the matched population without BAA. SRC and $B A A$ were found to be significantly associated with the presence of TAD (OR=2.57 and 7.69, respectively) and AAA $(\mathrm{OR}=2.81$ and 2.56 , respectively) on multivariable analysis. Conclusions This study establishes a substantial increased prevalence of aortic disease among patients with SRC and BAA. SRC and BAA should be considered markers for aortic aneurysm development.

\section{INTRODUCTION}

Thoracic aortic disease (TAD) and abdominal aortic aneurysms (AAA) are estimated to account for $1 \%-2 \%$ of all deaths in Western countries. ${ }^{1}$ These diseases are typically clinically silent until the potentially fatal complications of dissection or rupture develop. While screening recommendations exist to detect individuals with $\mathrm{AAAs}^{2}$ and thoracic aortic aneurysms (TAA), ${ }^{3}$ these measures are not adequate for identifying all patients with aortic disease. Ten to fifteen per cent of all AAAs present as a rupture, which is associated with a mortality rate of $50 \%-80 \%,{ }^{4}$ and aortic

\section{Key questions}

What is already known about this subject?

- Identifying clinical characteristics predictive of the development of thoracic aortic disease and abdominal aortic aneurysms would be valuable as these diseases are typically silent until potentially fatal complications develop.

- Prior studies have demonstrated an increased prevalence of simple renal cysts (SRC) and bovine aortic arch (BAA) among patients with aortic disease.

- The aim of our study, on the contrary, was to evaluate the prevalence of aortic disease among patients with SRC and BAA compared with a control population without these characteristics.

What does this study add?

- In a large population, this retrospective study establishes a substantial increased prevalence of thoracic aortic disease among patients with SRC and among patients with BAA beginning at age $>50$ and age $>30$, respectively, compared with those without these features.

- Additionally, this study demonstrates a significantly increased prevalence of abdominal aortic aneurysms among patients with SRC beginning at age $>40$ compared with those without SRC.

How might this impact on clinical practice?

- The study findings demonstrate that SRC and BAA should be considered as markers for aortic disease.

- Individuals with these characteristics may benefit from screening for aortic disease to hopefully prevent the devastating complications of aortic dissection and aneurysmal rupture.

dissection occurs in up to $60 \%$ of individuals at ascending aortic aneurysm size $<5.5 \mathrm{~cm},{ }^{5} \mathrm{a}$ commonly used threshold for surgical intervention. Moreover, $50 \%$ of patients with Stanford type A aortic dissection die before hospital admission. ${ }^{6}$ Therefore, identifying clinical characteristics predictive of the development of TAD and AAA would be valuable for detecting at-risk patients, as the mainstay of treatment is prophylactic surgical repair.

The main risk factors for AAA are cigarette smoking, advanced age, male sex and family 
history, ${ }^{7}$ while the major risk factors for TAD are hypertension, bicuspid aortic valve (BAV) and a family history/ genetic predisposition. ${ }^{8}$ Our group has introduced the paradigm 'Guilt by association' for the identification of TAAs, which uses various clinical markers linked to TAAs, including intracranial aneurysms, aortic arch anomalies, simple renal cysts (SRC), BAV, family history of aortic disease, a positive thumb-palm sign and temporal arteritis. ${ }^{9}$

Prior studies have demonstrated an increased prevalence of SRC and bovine aortic arch (BAA) among patients with aortic disease. ${ }^{10-16}$ However, in order to determine whether the presence of these characteristics in an asymptomatic patient is clinically actionable and should inform guidelines for screening, it is important to study the development of aortic disease in patients with these features. Therefore, the aim of our study was to evaluate the prevalence of aortic disease among patients with SRC and BAA compared with a control population without these characteristics. In essence, we are examining the converse of prior studies showing a higher incidence of SRC or BAA in patients with AAA or TAD.

\section{METHODS}

\section{Study population}

We performed a retrospective study of 35498 patients identified through an electronic medical record search from 2012 to 2016 for individuals who had both chest and abdominal CT imaging during that study period. This study included all patients at Yale-New Haven Hospital who fulfilled this criterion. Patients who underwent CT imaging both with and without contrast were included in the study. We used a number of different search terms to identify patients with TAD, AAA, SRC and BAA.

Baseline clinical characteristics for each patient were obtained and included age, sex and presence of several comorbidities (hypertension, hyperlipidaemia, diabetes mellitus (DM) and chronic kidney disease (CKD)). Exclusion criteria included patients who ${ }^{1}$ were $<18$ years of age, ${ }^{2}$ had end-stage renal disease (ESRD) or were on dialysis, ${ }^{3}$ had a diagnosis of polycystic kidney disease (PKD), or ${ }^{4}$ had a diagnosis of renal cell carcinoma (RCC)/malignant neoplasm of the kidney. Acquired cystic kidney disease has been shown to be highly prevalent among patients with $\mathrm{ESRD}^{17}$; hence, these patients were excluded. Additionally, cysts from PKD or RCC are not considered simple cysts, so these patients were also excluded. Individual patient consent for study inclusion was not obtained or required for this chart review study.

\section{Evaluation of radiologic imaging}

Only CT scans were used to determine the presence of TAD, AAA, SRC and BAA. We defined a patient as having a TAA if the ascending aorta was dilated to $\geq 4.0 \mathrm{~cm}$ or if the radiologist reported that the descending aorta was aneurysmal. While this lack of a defined cut-off for an aneurysmal descending aorta is a limitation of our study, there is no established cut-off for what is considered an aneurysmal descending aorta in the literature, given that the normal aortic diameter of the thoracic aorta is affected by a number of factors, such as age, sex and body size. ${ }^{3}$ We therefore used the experience of our chest radiologists in determining which descending aortas were considered aneurysmal. All patients recorded as having aortic aneurysms or dissections by the search were verified by our team to have aneurysms or dissections and the type of dissection and location of aneurysm was recorded. A patient was defined as having an AAA if the abdominal aorta was $\geq 3.0 \mathrm{~cm}$, consistent with published guidelines. ${ }^{18}$ A patient was considered to have an SRC if a round or oval low-attenuation lesion with thin walls and size $\geq 4 \mathrm{~mm}$ without obvious evidence of radiographic enhancement or septations was detected on abdominal CT, in accordance with our prior study. ${ }^{10}$ Lastly, a patient was considered to have a BAA if the innominate and left common carotid arteries shared a common origin or if the left common carotid branched directly off of the innominate artery.

\section{Statistical analysis}

Data are reported using means and SDs for continuous variables or as frequencies for categorical variables. Student's t-tests were used to analyse continuous variables. $\mathrm{X}^{2}$ tests were used to compare categorical variables. Multivariable logistic regression was performed to evaluate the association of patient characteristics, including age, sex, hypertension, hyperlipidaemia, DM, CKD, SRC and BAA, with aortic disease. Propensity score matching was employed for patients with SRC and for patients with BAA to identify controls matched by age, gender, hypertension, hyperlipidaemia, DM and CKD. Statistical analyses were performed using R V.3.1.0 (R Foundation for Statistical Computing, Vienna, Austria) and vassarstats. net.

\section{RESULTS}

\section{Overall patient characteristics}

We identified 35498 patients who had both a chest and abdominal CT scan between 2012 and 2016 (table 1) with a mean age of $60.4 \pm 18.0$ years. Among these patients, $6366(17.9 \%)$ had at least one SRC, $1355(3.8 \%)$ had a TAA, $253(0.7 \%)$ had an aortic dissection and $952(2.7 \%)$ had an AAA. Only 920 (2.6\%) were identified as having a BAA, which is substantially lower than the estimated prevalence of $15 \%-35 \%$ in the general US population. ${ }^{12}$ This indicates that radiologists are likely under-reporting the presence of BAAs on chest CTs.

\section{Prevalence of SRC}

The prevalence of SRC among patients with an ascending aortic aneurysm, descending aortic aneurysm, type A aortic dissection and type B aortic dissection was $44.9 \%$, $51.1 \%, 38.8 \%$ and $45.3 \%$, respectively. In all four groups, the prevalence of SRC was significantly higher than among patients who did not have any TAD (16.8\%). 


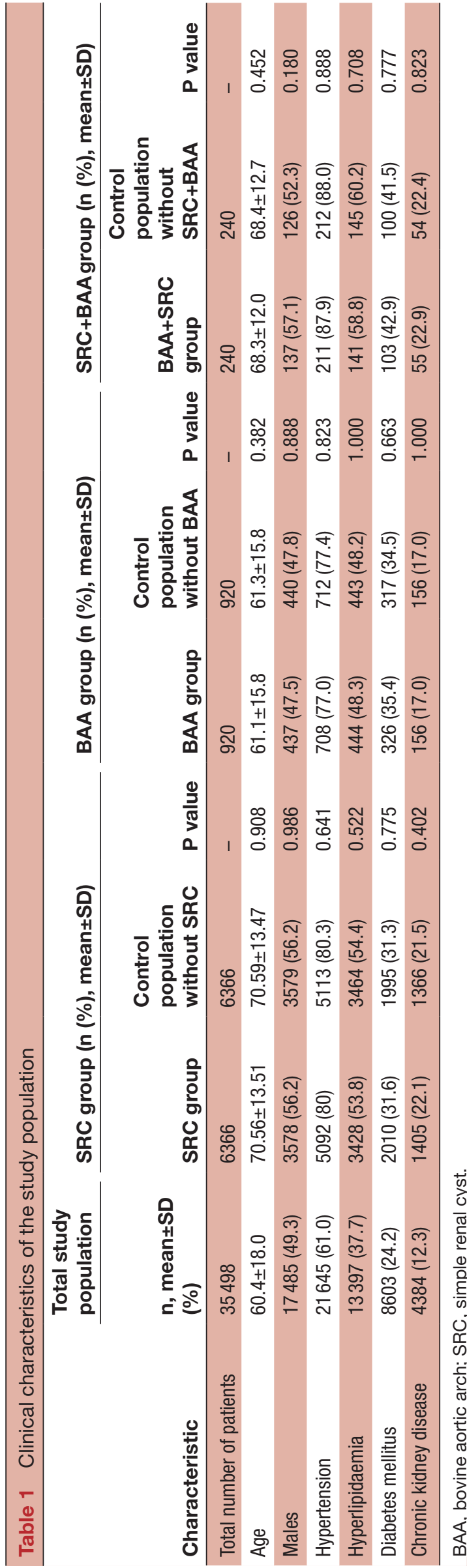

Additionally, the prevalence of SRC among patients with an AAA was significantly higher than that among patients who did not have an AAA (52.8\% vs $17.0 \%)$.

Prevalence of SRC was also calculated for all age groups (in 10 year age increments)) of patients with TAD, AAA and controls for each of these groups. We found that the prevalence of SRC was significantly higher among the TAD group versus the control group without TAD for each decade of life beginning at age 40. Similarly, there was a significantly increased prevalence of SRC among patients with AAA as compared with the control group without AAA for each decade of life beginning at age 50 .

\section{Prevalence of aortic disease among patients with SRC}

In order to examine the prevalence of aortic disease among patients with SRC, we performed propensity matching to generate a group of control patients without SRC. As shown in table 1, patients in the propensity matched control population did not differ significantly in age, sex or presence of comorbidities from the SRC group. Figure 1A and table 2 demonstrate that there is a significantly increased prevalence of all types of aortic disease in the SRC population compared with the control population. Moreover, for every decade of life starting in the sixth decade, there was a significantly increased prevalence of TAD among patients with SRC as compared with the propensity matched population without SRC (figure 1B). Additionally, for every decade of life starting in the fifth decade, there was a significantly increased prevalence of AAA among patients with SRC as compared with the propensity matched population without SRC (figure 1B).

\section{Prevalence of aortic disease among patients with BAA}

Since only $2.6 \%$ of the study population was reported as having a BAA in the CT radiology reports, it is likely that a substantial number of patients in the 'non-BAA' control group did indeed have a BAA. Therefore, we compared the prevalence of aortic disease among the BAA group with a propensity matched control population without BAA. The chest CT scans of the 920 patients in this control population were reanalysed to verify these patients indeed did not have a BAA.

The clinical characteristics of patients with BAA and without BAA in the propensity matched population are depicted in table 1. These groups of patients did not differ significantly in terms of age, sex or presence of comorbidities. However, patients with BAA were significantly more likely to have an SRC compared with the propensity matched control population.

Figure $2 \mathrm{~A}$ and table 2 illustrate that there is a significantly increased prevalence of all types of TAD in the BAA population compared with the control population. Furthermore, for every decade of life starting in the fourth decade, there was a significantly increased prevalence of TAD among patients with BAA as compared with the propensity matched population without BAA (figure 2B). 

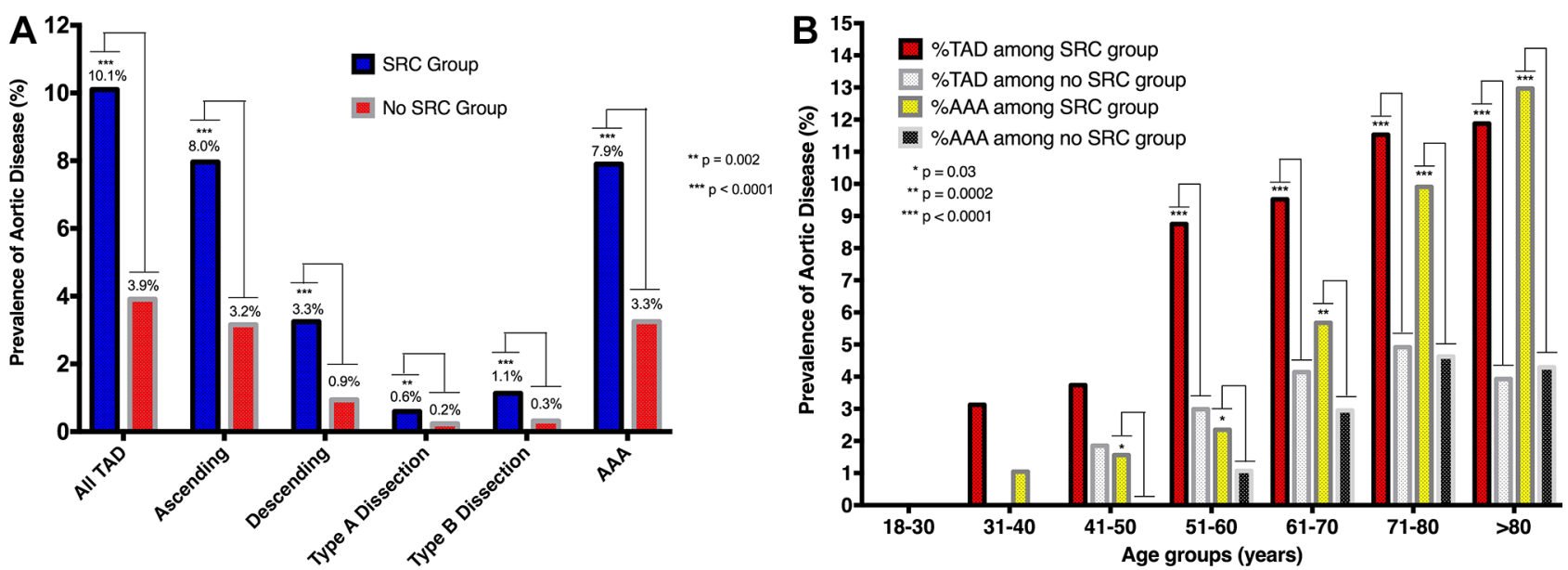

Figure 1 (A) Prevalence of aortic disease among propensity score matched patients with simple renal cyst versus without simple renal cyst. (B) Prevalence of aortic disease among propensity score matched patients with simple renal cyst versus without simple renal cyst by age group. AAA, abdominal aortic aneurysm; SRC, simple renal cyst; TAD, thoracic aortic disease.

\section{Prevalence of aortic disease among patients with BAA and SRC}

We also sought to determine the prevalence of TAD among patients with both BAA and SRC. Therefore, 240 patients with both BAA and SRC were propensity matched with 240 patients with neither feature. As table 1 demonstrates, these groups of patients did not differ significantly in terms of age, sex or presence of comorbidities. Figure 3 and table 2 demonstrate that there is a significantly increased prevalence of all types of TAD in the BAA+SRCpopulation compared with the control population except for type A aortic dissection.

\section{Multivariable logistic regression}

Multivariable logistic regression analysis was conducted to examine the association of SRC and BAA with aortic disease (table 3). SRC and BAA were each significantly associated with each type of aortic disease (table 3 and online supplementary table 1 ). Since the bovine arch status of the majority of patients in the study was unknown, we performed multivariable logistic regression analysis of the association of BAA with aortic disease both in the total study population and the population for which bovine status was known, which revealed similar results (online supplementary table 2 ).

As shown in table 3, on multivariable analysis, female sex and DM were both associated with a decreased risk of TAD. On the other hand, age, hypertension and hyperlipidaemia were all associated with an increased risk of TAD. CKD was also found to be significantly associated with AAA but not with TAD.

\section{Accuracy of search}

Since a variety of search terms were used to identify individuals with SRC and aortic aneurysms, we sought to determine the accuracy of the search by randomly selecting 1000 patients from the study population. Among these patients, our search missed $7 \%$ of SRC and $2.3 \%$ of aortic aneurysms (ie, a radiologist had described these findings in the radiology report but the search did not identify these patients as having these findings). Additionally, $0.4 \%$ of patients had CT scans that were never completed at Yale-New Haven Hospital. In these patients' electronic medical charts, it states that either the CT scan was ordered and never completed or it was completed at an outside hospital, so any search would not have identified these patients as having or not having aortic aneurysms or SRCs.

\section{DISCUSSION}

Prior research has demonstrated an increased prevalence of aortic disease among patients with $\mathrm{SRC}^{10} 1113$ and BAA. ${ }^{12} 14-16$ In a large cohort of patients with aortic disease (1355 with TAA and 253 with aortic dissection), we replicated our prior findings that there is an increased prevalence of SRC in patients with TAD. ${ }^{10}$ Just as in our prior study, we found the strongest association between the presence of SRC and TAD in patients with descending aortic aneurysms and type $\mathrm{B}$ dissection. We also replicated our findings that there is an increased prevalence of SRC among patients with TAD in each age group $>40$. Our study also establishes a threefold increased prevalence of SRC among patients with AAA (occurring among all age groups $>50$ ), which is similar to the 2.5 -fold increased prevalence of SRC among patients with AAA that Takagi and Umemoto demonstrated. ${ }^{13}$

In order to investigate whether SRC and BAA are risk factors for aortic disease, we sought to determine the prevalence of aortic disease in patients with these characteristics. After controlling for age, sex and several comorbidities (hypertension, hyperlipidaemia, DM and CKD) via propensity score matching, we found a significantly increased prevalence of all types of aortic disease in the SRC group compared with the group without SRC. In particular, we observed a significantly increased prevalence of TAD beginning in the sixth decade of life and AAA beginning in the fifth decade of life as compared with the propensity matched population. Among patients with BAA, there was a significantly increased prevalence 
of all forms of TAD as compared with a propensity matched population without BAA. This increased prevalence occurred as early as the fourth decade of life and persisted throughout all age groups.

Our multivariable analysis confirmed that SRC and BAA are risk factors for aortic disease. Compared with hypertension, an established risk factor for $\mathrm{TAD}^{8}$ and $\mathrm{AAA},{ }^{7}$ we found an equally strong or stronger association between SRC and TAD $(\mathrm{OR}=2.57)$ and between BAA and TAD $(\mathrm{OR}=7.69)$ than between hypertension and TAD $(\mathrm{OR}=2.25)$. Similarly, there was an equally strong or stronger association between $\mathrm{SRC}$ and AAA $(\mathrm{OR}=2.81)$ than between hypertension and AAA $(\mathrm{OR}=2.46)$. Among the different types of aortic disease, we observed that the strongest association with SRC was with descending aortic aneurysms $(\mathrm{OR}=3.09)$, while the strongest for BAA was with ascending aortic aneurysms $(\mathrm{OR}=8.25)$.

Our multivariable analysis findings of a negative association between $\mathrm{DM}$ and TAD $(\mathrm{OR}=0.57)$ and between $\mathrm{DM}$ and AAA $(\mathrm{OR}=0.66)$ are consistent with prior studies that have established a protective role for DM in the development of $\mathrm{TAD}^{19}$ and $\mathrm{AAA}^{20}$ Moreover, we observed a positive association between $\mathrm{CKD}$ and AAA $(\mathrm{OR}=1.21)$, which is consistent with a recent report. ${ }^{21}$ While CKD was not associated with TAD overall, it was significantly associated with descending aortic aneurysms $(\mathrm{OR}=1.43)$ (online supplementary table 1 ).

Prior studies have established that there is an increased risk for aortic dissection ${ }^{22}$ and $\mathrm{AAA}^{7}$ among males as compared with females, while the incidence of TAA has been demonstrated to be similar among the sexes. ${ }^{23}$ In our multivariable analysis, we found that female sex was negatively associated with both TAD $(\mathrm{OR}=0.48)$ and AAA $(\mathrm{OR}=0.44)$. Interestingly, however, when one analyzes the association between female sex and the type of TAD, female sex was only a statistically significant negative risk factor for aortic disease among individuals with ascending aortic aneurysms $(\mathrm{OR}=0.39)$ and type $\mathrm{A}$ aortic dissection $(\mathrm{OR}=0.60)$ but not for descending aortic aneurysms or type $\mathrm{B}$ aortic dissection (online supplementary table 1 ).

For the features in our 'Guilt by Association' paradigm, studies have demonstrated that individuals with intracranial aneurysms, ${ }^{24}$ AAA, ${ }^{25}$ giant cell arteritis ${ }^{26}$ and $\mathrm{BAV}^{27}$ have higher rates of TAAs than individuals without these features. However, no study has analysed the prevalence of TAD among individuals with more than one of these features. Therefore, we compared the prevalence of TAD among 240 patients with both SRC and BAA with a propensity matched population with neither of these features and found that these patients were even more likely to have TAD than those with either SRC alone or BAA alone $(32.1 \%$ vs $10.1 \%$ and $21.8 \%$, respectively). This finding indicates that perhaps one can use the 'Guilt by Association' paradigm as a stepwise ladder, in which individuals with multiple features have an even greater risk for TAD than those with simply one feature. 

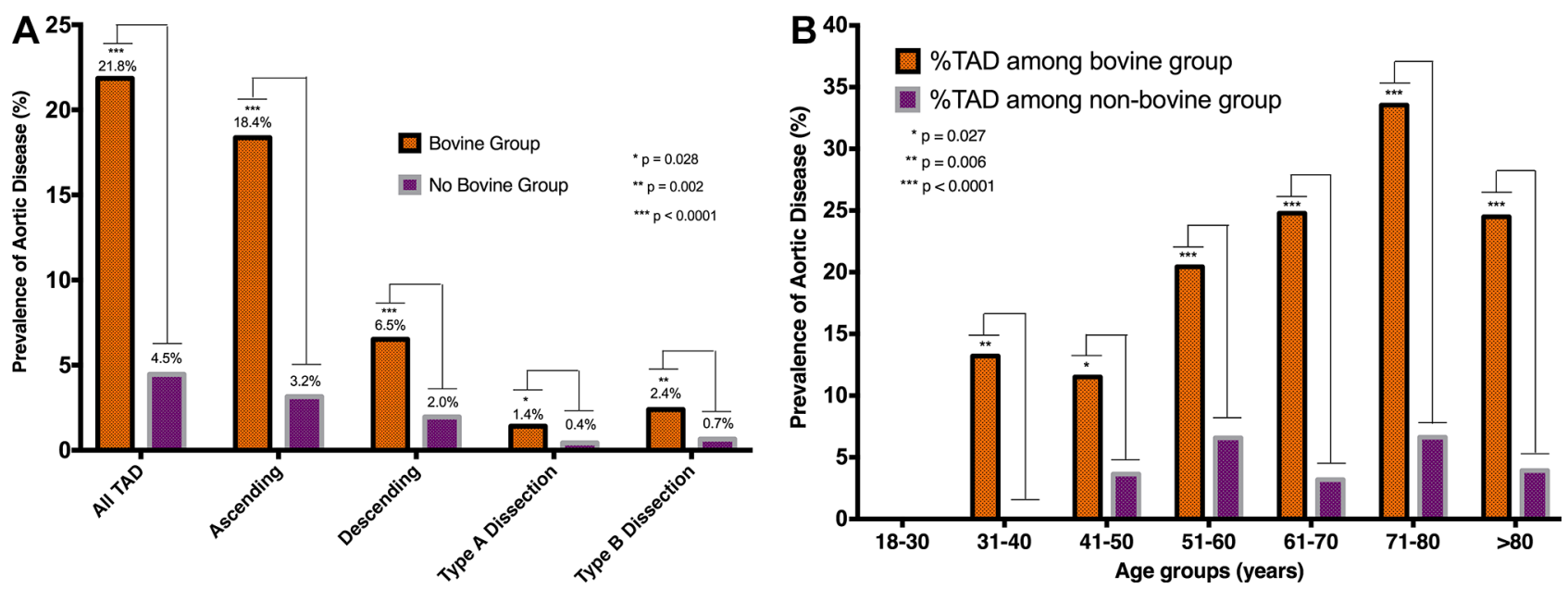

Figure 2 (A) Prevalence of thoracic aortic disease among propensity score matched patients with bovine aortic arch versus no bovine aortic arch. (B) Prevalence of thoracic aortic disease among propensity score matched patients with bovine aortic arch versus no bovine aortic arch by age group. TAD, thoracic aortic disease.

The limitations of our study include its retrospective nature. Therefore, it is difficult to establish SRC and BAA as definitive risk factors for TAD and AAA without performing a prospective study. Furthermore, the search that was performed was not entirely accurate since we found that $7 \%$ of SRC and $2.3 \%$ of aortic aneurysms were missed. However, this does not alter the fact that there is a significant association between SRC and aortic disease. In fact, since $7 \%$ of patients with SRC were missed and were therefore included in the control population, our analysis, if anything, underestimates the true association between SRC and aortic disease. Furthermore, we were not able to control for all risk factors associated with aortic disease in our propensity matching analysis, including smoking history, family history and presence of connective tissue disorders.

It is worth contemplating exactly how the evidence from this paper should be applied to clinical practice.

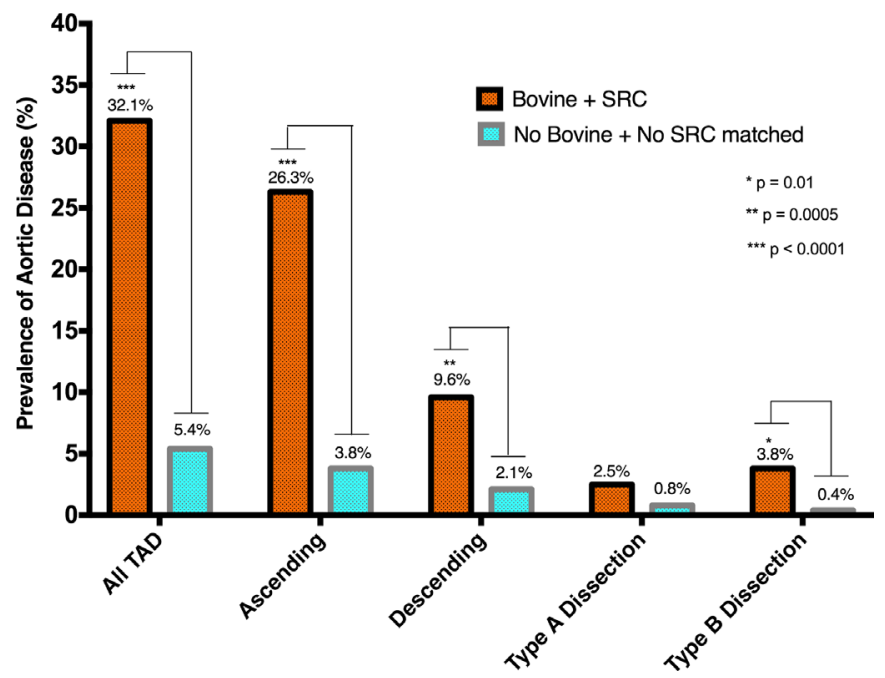

Figure 3 Prevalence of thoracic aortic disease among patients with bovine aortic arch and simple renal cyst versus patients with neither. SRC, simple renal cyst; TAD, thoracic aortic disease.
We can consider first the patient with BAA. The BAA will have been diagnosed on a three-dimensional imaging study (usually CT scan, but possibly MRI). An associated TAA may be evident at that time on that very study. If not, the awareness of the BAA and its link with TAA should lead to periodic surveillance imaging.

We can consider next the patient with SRC. The SRC will usually have been diagnosed on a CT of the abdomen. Consideration should be given to extending the CT scan to include the chest (just a few more frames above the standard abdominal CT scan). If the chest is negative at that time, periodic imaging surveillance should be considered in the overall clinical context.

For both BAA and SRC, transthoracic cardiac ECHO (to visualise the ascending aorta) and abdominal ultrasound, as well as MRI of the chest and/or abdomen, can be invoked as needed in order to limit somatic radiation exposure. The clinical implications for patients diagnosed with BAA or SRC stated above require further study, including cost-effectiveness analyses, before any final recommendations for screening can be advocated.

We feel that the 'guilt by association' demonstrated in this study-specifically that BAA and SRC are often seen together with TAD and AAA—can be exploited to identify otherwise silent aneurysm disease and in this way save lives that could otherwise be lost to dissection or rupture.

\section{CONCLUSION}

In this retrospective study, we established a substantial increased prevalence of TAD among patients with SRC and among patients with BAA beginning at age $>50$ and age $>30$, respectively. We also found an increased prevalence of AAAs among patients with SRCs beginning at age $>40$. These findings indicate that SRCs and BAA should be considered as markers for the development of aortic disease. 
Table 3 Multivariable logistic regression examining the association of patient characteristics with aortic disease

\begin{tabular}{|c|c|c|c|c|c|c|}
\hline \multirow[b]{2}{*}{ Variable } & \multicolumn{3}{|c|}{ Thoracic aortic disease } & \multicolumn{3}{|c|}{ Abdominal aortic aneurysm } \\
\hline & OR & $95 \% \mathrm{Cl}$ & $P$ value & OR & $95 \% \mathrm{Cl}$ & $P$ value \\
\hline SRC & 2.57 & 2.29 to 2.88 & $4.2 \times 10^{-57}$ & 7.33 & 5.01 to 10.98 & $1.8 \times 10^{-23}$ \\
\hline BAA & 7.33 & 5.01 to 10.98 & $1.8 \times 10^{-23}$ & 2.95 & 1.7 to 5.2 & 0.00009 \\
\hline Female sex & 0.48 & 0.43 to 0.54 & $3.3 \times 10^{-35}$ & 0.44 & 0.39 to 0.51 & $4.3 \times 10^{-29}$ \\
\hline Age & 1.02 & 1.02 to 1.03 & $6.1 \times 10^{-28}$ & 1.05 & 1.05 to 1.06 & $6.7 \times 10^{-68}$ \\
\hline Hypertension & 2.25 & 1.91 to 2.65 & $3.0 \times 10^{-22}$ & 2.46 & 1.96 to 3.13 & $3.7 \times 10^{-14}$ \\
\hline Hyperlipidaemia & 1.33 & 1.18 to 1.50 & 0.0000029 & 1.78 & 1.53 to 2.06 & $5.3 \times 10^{-14}$ \\
\hline Diabetes mellitus & 0.57 & 0.50 to 0.65 & $3.1 \times 10^{-16}$ & 0.66 & 0.56 to 0.76 & $8.8 \times 10^{-8}$ \\
\hline Chronic kidney disease & 1.15 & 0.995 to 1.32 & 0.056 & 1.21 & 1.03 to 1.41 & 0.02 \\
\hline
\end{tabular}

BAA, bovine aortic arch; SRC, simple renal cyst.

\section{Author affiliations}

${ }^{1}$ Aortic Institute at Yale-New Haven Hospital, Yale School of Medicine, New Haven, Connecticut, USA

${ }^{2}$ Department of Medicine, Johns Hopkins Hospital and Johns Hopkins School of Medicine, Baltimore, MD, USA

${ }^{3}$ Department of Political Science and Economics, Rowan University, Glassboro, New Jersey, USA

${ }^{4}$ Department of Economics, Stony Brook University, Stony Brook, New York, USA ${ }^{5}$ Department of Family, Population and Preventive Medicine, Stony Brook University, Stony Brook, New York, USA

${ }^{6}$ Section of Nephrology, Department of Internal Medicine, Yale University School of Medicine, New Haven, Connecticut, USA

${ }^{7}$ Section of Vascular and Endovascular Surgery, Yale School of Medicine, New Haven, Connecticut, USA

${ }^{8}$ Department of Surgical Diseases № 2, Kazan State Medical University, Kazan, Russia

Contributors AJB, SUBM, AS, CVM, MAZ, NKD, YE, BAZ and JAE all contributed to the planning, conduct and reporting of the work described in the article. YL and JAR contributed to the statistical analysis of the reported data. AJB and JAE are responsible for the overall content as guarantors.

Funding The authors have not declared a specific grant for this research from any funding agency in the public, commercial or not-for-profit sectors.

Competing interests None declared.

Patient consent for publication Not required.

Ethics approval Yale Institutional Review Board.

Provenance and peer review Not commissioned; externally peer reviewed.

Open access This is an open access article distributed in accordance with the Creative Commons Attribution Non Commercial (CC BY-NC 4.0) license, which permits others to distribute, remix, adapt, build upon this work non-commercially, and license their derivative works on different terms, provided the original work is properly cited, appropriate credit is given, any changes made indicated, and the use is non-commercial. See: http://creativecommons.org/licenses/by-nc/4.0/.

\section{REFERENCES}

1. Verstraeten A, Luyckx I, Loeys B. Aetiology and management of hereditary aortopathy. Nat Rev Cardiol 2017;14:197-208.

2. Lee ES, Pickett E, Hedayati N, et al. Implementation of an aortic screening program in clinical practice: implications for the Screen For Abdominal Aortic Aneurysms Very Efficiently (SAAAVE) Act. $J$ Vasc Surg 2009;49:1107-11.

3. Hiratzka LF, Bakris GL, Beckman JA, et al. 2010 ACCF/AHA/AATS/ ACR/ASA/SCA/SCAI/SIR/STS/SVM Guidelines for the diagnosis and management of patients with thoracic aortic disease. A Report of the American College of Cardiology Foundation/American Heart Association Task Force on Practice Guidelines, American Association for Thoracic Surgery, American College of Radiology,American Stroke Association, Society of Cardiovascular Anesthesiologists,
Society for Cardiovascular Angiography and Interventions, Society of Interventional Radiology, Society of Thoracic Surgeons, and Society for Vascular Medicine. J Am Coll Cardiol 2010;55:e27-e129.

4. Kuivaniemi H, Ryer EJ, Elmore JR, et al. Update on abdominal aortic aneurysm research: from clinical to genetic studies. Scientifica 2014:2014:1-14.

5. Pape LA, Tsai TT, Isselbacher EM, et al. Aortic diameter $>$ or $=5.5$ $\mathrm{cm}$ is not a good predictor of type A aortic dissection: observations from the International Registry of Acute Aortic Dissection (IRAD). Circulation 2007;116:1120-7.

6. Howard DP, Banerjee A, Fairhead JF, et al. Population-based study of incidence and outcome of acute aortic dissection and premorbid risk factor control: 10-year results from the Oxford Vascular Study. Circulation 2013;127:2031-7.

7. Kuivaniemi HR, Yoon EJ, Elmore HR, et al. Genetic risk factors for abdominal Aortic Aneurysms (AAA). Bennington E, ed. Horizons in world cardiovascular research series. Hauppauge, NY: Nova Science Publishers, 2015:1-31.

8. Milewicz D, Hostetler E, Wallace S, et al. Precision medical and surgical management for thoracic aortic aneurysms and acute aortic dissections based on the causative mutant gene. J Cardiovasc Surg 2016;57:172-7.

9. Ziganshin BA, Elefteriades JA. Guilt by association: a paradigm for detection of silent aortic disease. Ann Cardiothorac Surg 2016;5:174-87.

10. Ziganshin BA, Theodoropoulos $\mathrm{P}$, Salloum MN, et al. Simple renal cysts as markers of thoracic aortic disease. J Am Heart Assoc 2016;5.

11. Kim EK, Choi ER, Song BG, et al. Presence of simple renal cysts is associated with increased risk of aortic dissection: a common manifestation of connective tissue degeneration? Heart 2011;97:55-9.

12. Moorehead PA, Kim AH, Miller CP, et al. Prevalence of bovine aortic arch configuration in adult patients with and without thoracic aortic pathology. Ann Vasc Surg 2016;30:132-7.

13. Takagi H, Umemoto T. ALICE (All-Literature Investigation of Cardiovascular Evidence) Group. Simple renal cyst and abdominal aortic aneurysm. J Vasc Surg 2016;63:254-9.

14. Wanamaker KM, Amadi CC, Mueller JS, et al. Incidence of aortic arch anomalies in patients with thoracic aortic dissections. J Card Surg 2013;28:151-4.

15. Malone CD, Urbania TH, Crook SE, et al. Bovine aortic arch: a novel association with thoracic aortic dilation. Clin Radiol 2012;67:28-31.

16. Hornick M, Moomiaie R, Mojibian $\mathrm{H}$, et al. 'Bovine' aortic arch - a marker for thoracic aortic disease. Cardiology 2012;123:116-24.

17. Dunnill MS, Millard PR, Oliver D. Acquired cystic disease of the kidneys: a hazard of long-term intermittent maintenance haemodialysis. J Clin Pathol 1977;30:868-77.

18. Hirsch AT, Haskal ZJ, Hertzer NR, et al. ACC/AHA 2005 Practice Guidelines for the management of patients with peripheral arterial disease (lower extremity, renal, mesenteric, and abdominal aortic): a collaborative report from the American Association for Vascular Surgery/Society for Vascular Surgery, Society for Cardiovascular Angiography and Interventions, Society for Vascular Medicine and Biology, Society of Interventional Radiology, and the ACC/AHA Task Force on Practice Guidelines (Writing Committee to Develop Guidelines for the Management of Patients With Peripheral Arteria Disease): endorsed by the American Association of Cardiovascular 
and Pulmonary Rehabilitation; National Heart, Lung, and Blood Institute; Society for Vascular Nursing; TransAtlantic Inter-Society Consensus; and Vascular Disease Foundation. Circulation 2006;113:e463-654

19. Takagi H, Umemoto T. ALICE (All-Literature Investigation of Cardiovascular Evidence) Group. Negative association of diabetes with thoracic aortic dissection and aneurysm. Angiology 2017;68:216-24.

20. De Rango P, Farchioni L, Fiorucci B, et al. Diabetes and abdominal aortic aneurysms. Eur J Vasc Endovasc Surg 2014;47:243-61.

21. Takeuchi H, Okuyama M, Uchida HA, et al. Chronic kidney disease is positively and diabetes mellitus is negatively associated with abdominal aortic aneurysm. PLoS One 2016;11:e0164015.

22. LeMaire SA, Russell L. Epidemiology of thoracic aortic dissection. Nat Rev Cardiol 2011;8:103-13.
23. Clouse WD, Hallett JW, Schaff HV, et al. Improved prognosis of thoracic aortic aneurysms: a population-based study. JAMA 1998;280:1926-9.

24. Kuzmik GA GM, Bulsara KR, Tranquilli M, et al. Intracranial aneurysm patients may harbor thoracic aortic aneurysms. 2012. Available online http://aatsorg/aortic/abstracts/2012/303cgi

25. Larsson E, Vishnevskaya L, Kalin B, et al. High frequency of thoracic aneurysms in patients with abdominal aortic aneurysms. Ann Surg 2011:253:180-4.

26. Mackie SL, Hensor EM, Morgan AW, et al. Should I send my patient with previous giant cell arteritis for imaging of the thoracic aorta? A systematic literature review and meta-analysis. Ann Rheum Dis 2014;73:143-8.

27. Verma S, Siu SC. Aortic dilatation in patients with bicuspid aortic valve. N Engl J Med 2014;370:1920-9. 